\title{
Spin Supersolid in Anisotropic Spin-One Heisenberg Chain
}

\author{
P. Sengupta ${ }^{1,2}$ and C. D. Batista ${ }^{1}$ \\ ${ }^{1}$ Theoretical Division, Los Alamos National Laboratory, Los Alamos, NM 87545 \\ ${ }^{2}$ MST-NHMFL, Los Alamos National Laboratory, Los Alamos, NM 87545
}

(Dated: March 23, 2022)

\begin{abstract}
We consider an $S=1$ Heisenberg chain with strong exchange $(\Delta)$ and single-ion uniaxial anisotropy $(D)$ in a magnetic field $(B)$ along the symmetry axis. The low energy spectrum is described by an effective $S=1 / 2$ $\mathrm{XXZ}$ model that acts on two different low energy sectors for a given window of fields. The vacuum of each sector exhibits Ising-like antiferromagnetic ordering that coexists with the finite spin stiffness obtained from the exact solution of the effective XXZ model. In this way, we demonstrate the existence of a spin supersolid phase. We also compute the full $\Delta-B$ quantum phase diagram by means of a quantum Monte Carlo simulation.
\end{abstract}

PACS numbers: 75.10.Jm, 75.40.Mg, 75.40.Cx

One of the primary goals in the study of strongly correlated systems is the search for novel states of matter. These novel states usually emerge from competing interactions, each of which tends to stabilize distinct orderings. Different outcomes can result from such competition: a) none of the competing phases prevail, b) one interaction becomes dominant at low energies and stabilizes the corresponding ordering, c) more than one competing orderings coexist in a new phase. In the last case, the coexistence can be homogeneous or inhomogeneous. The supersolid phase is one of the simplest examples of homogeneous coexistence of two different phases (solid and superfluid) in bosonic systems. However, this novel state has been elusive both from an experimental [1] and a theoretical [2] viewpoint. While it is still unclear whether a supersolid (SS) state can be stabilized in the continuum, there are several numerical studies which show that a SS phase can be stabilized in the presence of a periodic potential or underlying lattice [3, 4, 5]. Surprisingly, this numerical effort has not been complemented whatsoever with rigorous analytical treatments that can demonstrate the existence of the SS phase, at least in certain limits.

The SS state is easier to stabilize on a lattice because the lattice parameter of the "solid phase" or charge density wave cannot relax to any arbitrary value (it has to be an integer multiple of the underlying lattice parameter). There are two natural realizations of bosonic gases on a lattice: atomic gases in optical lattices or periodic substrates and quantum magnets (spin lattices). Although most of the previous numerical work has been focused on the former systems [3, 4, 5], we have shown recently [6] that a class of spin Hamiltonians, which describe real magnets to a very good approximation, also contain a SS phase in their quantum phase diagram. In particular, the models for hard core bosons on frustrated lattices that have been considered in the context of atomic gases can also be generated as low energy effective theories of frustrated spindimer systems [6]. The interest and the number of numerical works on spin SS phases is rapidly increasing [7, 8, 9].

The area of 1D magnetism has also attracted a lot of attention during the last few decades. This interest was mainly triggered by the successful application of field theory techniques for finding relevant and solvable low energy effective theo- ries. A remarkable accomplishment was achieved by Haldane [10], who pointed out a qualitative difference between the low energy spectra of integer and half-odd-integer Heisenberg chains. The gapped Haldane phase has peculiar properties with measurable consequences, like the $S=1 / 2$ endchain states of open $S=1$ Heisenberg chains [11].

In spite of the intense effort devoted to the study of 1D Heisenberg like spin models, the search for new states and behaviors is far from being complete. In this Letter, we demonstrate that the low energy spectrum of an $S=1$ Heisenberg chain with uniaxial exchange and single-ion anisotropy consists of two sectors, each of which can be mapped into the exactly solvable $(S=1 / 2)$ XXZ model for the limit of strong anisotropy. By exploiting this mapping, we also demonstrate that the ground state is a SS (Ising-like spin ordering coexisting with a finite spin stiffness) in a finite range of magnetic field. This is a surprising result considering that 1D solid phases are usually unstable when the particle density (magnetization for spin systems) deviates from the corresponding commensurate value. This occurs because each added (or removed) particle introduces a soliton in the ground state, something that is particularly clear in the bosonization treatments [12]. In our case, the solid (Ising) phase stabilized by the exchange anisotropy and the solitons remain as massive excitations for a finite range of densities due to the presence of a significant single ion anisotropy. This exceptional behavior leads to the stabilization of the supersolid state. In addition, we compute the full quantum phase diagram of $H$ that covers different regimes of anisotropy and was computed using a quantum Monte Carlo (QMC) algorithm.

We start by considering an $S=1$ Heisenberg model with uniaxial exchange and single-ion anisotropies on a linear chain $\mathcal{L}$ of $L$ sites:

$$
H=\sum_{i=1, L} J_{\perp}\left(S_{i}^{x} S_{i+1}^{x}+S_{i}^{y} S_{i+1}^{y}\right)+J_{z} S_{i}^{z} S_{i+1}^{z}+D S_{i}^{z 2}-B S_{i}^{z} .
$$

Here $J_{z}>0, D>0$ and we define $L+1 \equiv 1$ to impose periodic boundary conditions (PBC). Since $H$ is invariant under spin-rotations along the $z$-axis, the $z$-component of the magnetization, $M=\sum_{i} S_{i}^{z}$, is a good quantum number. We will assume that $\Delta=J_{z} / J_{\perp} \gg 1, J_{\perp} \ll D, J_{\perp} \ll J_{z}-D$ and 
$J_{z}>D$. Therefore, we can treat $J_{\perp}$ as a perturbation. For $J_{\perp}=0, H$ becomes diagonal in the basis of eigenstates of the set of operators $\left\{S_{i}^{z}\right\}$. The chain system consists of two interpenetrating sublattices $\mathcal{A}$ and $\mathcal{B}$. Since $J_{z}>D>0$, there are only two ground states for $B=0$ (i.e., $M=0$ ): $S_{i}^{z}= \pm 1$ for $i \in \mathcal{A}$ and $S_{i}^{z}=\mp 1$ for $i \in \mathcal{B}$ :

$$
\begin{aligned}
\left|\psi_{\mathcal{A}}^{0}\right\rangle & =\frac{1}{2^{L / 2}} \prod_{i \in \mathcal{A}} S_{i}^{+} \prod_{i \in \mathcal{B}} S_{i}^{-}|00 \ldots . .0\rangle \\
\left|\psi_{\mathcal{B}}^{0}\right\rangle & =\frac{1}{2^{L / 2}} \prod_{i \in \mathcal{B}} S_{i}^{+} \prod_{i \in \mathcal{A}} S_{i}^{-}|00 \ldots . .0\rangle
\end{aligned}
$$

where $|00 \ldots . .0\rangle$ is the reference state in which all the spins are in the eigenstate of $S_{i}^{z}$ with eigenvalue zero: $S_{i}^{z}|00 \ldots . .0\rangle=0$ $\forall i \in \mathcal{L}$. Any other state with $M=0$ has an energy $2 J_{z}-D$ or higher. Let us consider now the case $0<M<L / 2$. In this case, the low energy subspace can again be divided into two different sectors or subspaces $\mathcal{S}^{\mathcal{A}}$ and $\mathcal{S}^{\mathcal{B}}$. The subspace $\mathcal{S}^{A}$ is generated by the following basis of states:

$$
\left\{\left|\phi_{i_{1} \ldots i_{M}}^{\mathcal{A}}\right\rangle=\frac{1}{2^{M / 2}} S_{i_{1}}^{+} S_{i_{2}}^{+} \ldots S_{i_{M}}^{+}\left|\psi_{\mathcal{A}}\right\rangle\right\}
$$

where the sites $\left\{i_{1} \ldots i_{M}\right\} \in \mathcal{B}$ are all different: $i_{1}<i_{2} \ldots<$ $i_{M}$. In the same way, the subspace $\mathcal{S}^{\mathcal{B}}$ is generated by the basis of states:

$$
\left\{\left|\phi_{j_{1} \ldots j_{M}}^{\mathcal{B}}\right\rangle=\frac{1}{2^{M / 2}} S_{j_{1}}^{+} S_{j_{2}}^{+} \ldots . S_{j_{M}}^{+}\left|\psi_{\mathcal{B}}\right\rangle\right\}
$$

where the sites $\left\{j_{1} \ldots j_{M}\right\} \in \mathcal{A}$ are all different: $j_{1}<j_{2} \ldots<$ $j_{M}$. The energy of any other state that has magnetization $M$ and is orthogonal to $\mathcal{S}=\mathcal{S}^{\mathcal{A}} \oplus \mathcal{S}^{\mathcal{B}}$ is higher by at least $2 J_{z}-D$. Therefore, we can use degenerate perturbation theory to solve the low energy spectrum for small $J_{\perp}$ : $J_{\perp} \ll 2 J_{z}-D$ and $J_{\perp} \ll D$. The subspaces $\mathcal{S}^{\mathcal{A}}$ and $\mathcal{S}^{\mathcal{B}}$ cannot be connected by any finite order process in the thermodynamic limit $L \rightarrow \infty$. Consequently, we have two identical and disconnected low energy theories on each sector. From now on, we will consider one of them without loss of generality. The low energy subspace $\mathcal{S}^{\mathcal{A}}(\mathrm{Eq} 3$ can be mapped into the Hilbert space for $M$ hard core bosons on the $L / 2$ sites of the $\mathcal{B}$ sublattice:

$$
\left|\phi_{i_{1} \ldots i_{M}}^{\mathcal{A}}\right\rangle=b_{i_{1}}^{\dagger} b_{i_{2}}^{\dagger} \ldots . b_{i_{N}}^{\dagger}\left|0_{\mathcal{A}}\right\rangle
$$

where $i_{1}<i_{2} \ldots i_{N} \in \mathcal{B}$. The relation between the hard core bosons and the original spin operators is given by:

$$
S_{i}^{+}=\sqrt{2} b_{i}^{\dagger}, \quad S_{i}^{-}=\sqrt{2} b_{i}, \quad S_{i}^{z}=-1+n_{i},
$$

where $i \in \mathcal{B}$ and $n_{i}=b_{i}^{\dagger} b_{i}$. We emphasize that these relations are only valid within the low energy subspace $\mathcal{S}^{\mathcal{A}}$. In addition, $S_{i}^{z}=1$ for $i \in \mathcal{A}$, which implies that the two-spin correlators $\left\langle S_{i}^{z} S_{j}^{z}\right\rangle$ and $\left\langle S_{i}^{+} S_{j}^{-}\right\rangle$have the following expressions up to quadratic corrections in the perturbative parameter $J_{\perp} / J_{z}$ :

$$
\begin{aligned}
\left\langle S_{j}^{z} S_{j+r}^{z}\right\rangle & =e^{i \pi r}\left(1-\left\langle n_{j}\right\rangle-\left\langle n_{j+r}\right\rangle\right)+\left\langle n_{j} n_{j+r}\right\rangle \\
\left\langle S_{j}^{+} S_{j+r}^{-}\right\rangle & =2\left\langle b_{j}^{\dagger} b_{j+r}\right\rangle
\end{aligned}
$$

where $b_{i}^{\dagger} \equiv 0$ and $n_{i} \equiv 0 \forall i \in \mathcal{A}$. After doing a canonical transformation and projecting out the high energy states:

$$
\tilde{H}=P e^{-S} H e^{s} P=\tilde{H}_{\mathcal{A}}+\tilde{H}_{\mathcal{B}}
$$

we obtain the following expression for the low energy effective model, $\tilde{H}_{\mathcal{A}}$, that acts on the sector $\mathcal{S}^{\mathcal{A}}$ :

$$
\tilde{H}_{\mathcal{A}}=L C+\sum_{i \in \mathcal{B}} t\left(b_{i}^{\dagger} b_{i+2}+b_{i+2}^{\dagger} b_{i}\right)-\mu n_{i}+V n_{i} n_{i+2}
$$

where $L+2 \equiv 2(\mathrm{PBC})$ and

$$
\begin{aligned}
C & =-\frac{J_{\perp}^{2}}{3 J_{z}-2 D}, \quad t=-\frac{J_{\perp}^{2}}{2\left(J_{z}-D\right)}, \\
\mu & =\frac{J_{\perp}^{2}}{J_{z}}-2 t+4 C+B+D-2 J_{z}, \\
V & =-\frac{J_{\perp}^{2}}{J_{z}}-2 t+2 C .
\end{aligned}
$$

We note that $|V|<-2 t$. $\tilde{H}_{\mathcal{A}}$ is the so-called $t-V$ model (or $\mathrm{S}=1 / 2 \mathrm{XXZ}$ Hamiltonian [14]) and is exactly solvable by the Bethe Ansatz method [13]. The ground state is a Luttinger liquid for $|V|<|2 t|$ and $-2|t|<\mu<2 V+2|t|$. The asymptotic behavior of the transverse and longitudinal two point correlators can be obtained with the bosonization method [12]:

$$
\begin{aligned}
\left\langle n_{j} n_{j+r}\right\rangle & =\tilde{\rho}^{2}-\frac{K}{2 \pi^{2} r^{2}}+C_{1} r^{-2 K} \cos (2 \pi \tilde{\rho} r) \\
\left\langle b_{j}^{\dagger} b_{j+r}\right\rangle & =C_{2} r^{-g(K)} \cos \alpha r+C_{3} r^{-1 / 2 K} \cos \pi r
\end{aligned}
$$

where $g(K)=2 K+1 /(2 K), \alpha=[(2 \tilde{\rho}-1) \pi, K$ is the Luttinger liquid (LL) parameter and $\tilde{\rho}=\left\langle n_{j}\right\rangle \forall i \in \mathcal{B}$. A schematic contour map of $K$ as a function of $V / t$ and $\tilde{\rho}$ can be found in Ref.[12]. The wave-length, $\lambda$, of the oscillations of the density-density correlator is the mean separation between bosons, i.e., $\lambda=1 / \tilde{\rho}$. Therefore, the longitudinal spin-spin correlator has two oscillatory components. The first component (first term of Eq7) has a constant amplitude and the wave-length is equal to two lattice parameters. This contribution comes from the "solid" or Ising component of the SS phase. The second contribution (second term of Eq 7) comes from the LL component and consequently decays with a power law according to $\mathrm{Eq} 12$. The density of the LL in the supersolid phase $(\rho \equiv \tilde{\rho} / 2$ since $\tilde{\rho}$ the density on the $\mathcal{B}$ sublattice) can be extracted from the wave-length, $2 \lambda$, of this oscillatory component.

In order to test the accuracy of our effective model, we used the LANCZOS method to compute the exact ground state of the original Hamiltonian, $H$, in a $L=16$ sites chain. Fig 1 a shows a comparison between the magnetization as function of field, $M(B)$, obtained with the original and the effective models for $J_{z}=20 J_{\perp}$ and $D=10 J_{\perp}$. The curves are practically indistinguishable. The full line is the $M(B)$ curve obtained with $\tilde{H}_{\mathcal{A}}$ in the thermodynamic limit $L \rightarrow \infty$. Fig 1 p shows a similar comparison for the field dependence of the longitudinal spin structure factor:

$$
S^{z z}(q)=\frac{1}{L} \sum_{j, k} e^{-i q(j-k)}\left\langle S_{j}^{z} S_{k}^{z}\right\rangle
$$


Again, practically the same curves are obtained with $H$ and $\tilde{H}_{\mathcal{A}}$ for a 16 sites chain. The finite value of $S^{z z}(q) / L$ for $L \rightarrow$ $\infty$ indicates the existence of Ising ordering. Finally, Fig 1 ; shows the same comparison for the field dependence of the spin stiffness $\rho_{s}$ or superfluid density in the bosonic language. To define $\rho_{s}$, we will use units of $\hbar=1$, lattice parameter $a=1$ and effective mass of the bosons $m=1 / 8 t=1$. $\rho_{s}$ can be defined as the response to the gauge field generated by an infinitesimal flux $\phi$ threading the ring (chain with PBC), $\rho_{s}=\frac{\partial^{2} \epsilon}{\partial^{2} \phi}$, where $\epsilon$ is the free energy per site (ground state energy density at $T=0$ ). According to Figs $1 \mathrm{p}$ and 1, the SS phase appears for $B_{c 1}<B<B_{c 2}$, where

$$
\begin{aligned}
& B_{c 1}=4 t+2 J_{z}-D-4 C-\frac{J_{\perp}^{2}}{J_{z}} \\
& B_{c 2}=2 V+2 J_{z}-D-4 C-\frac{J_{\perp}^{2}}{J_{z}}
\end{aligned}
$$

are determined by the conditions $\mu_{c 1}=2 t$ and $\mu_{c 2}=2 \mathrm{~V}-2 t$. Two different Ising phases appear on both sides: IS1 for $B<$ $B_{c 1}$ and IS2 for $B_{c 2}<B$. In the dilute limit, $B \rightarrow B_{c 1}^{+}$(see Fig. 17), the superfluid density approaches the value of the total density $\rho$ at $T=0$. This is the known result for bosons in the continuum that is recovered in the dilute limit of the lattice system $(\rho a \rightarrow 0)$.

The Ising order IS2 corresponds to the state in which $S_{i}^{z}=1 \forall i \in \mathcal{A}$ and $S_{i}^{z}=0 \forall i \in \mathcal{B}$. If we keep increasing the field, ground states with higher values of $M$ will be eventually stabilized. For $L / 2 \leq M<L$, we can again identify a low energy subspace generated by the states: $\left\{\left|\psi_{i_{1} \ldots i_{M}}\right\rangle=\frac{1}{2^{M / 2}} S_{i_{1}}^{+} S_{i_{2}}^{+} \ldots S_{i_{M}}^{+}|00 \ldots 0\rangle\right\}$ where $i_{n}$ denotes now any site of $\mathcal{L}$ and $i_{1}<i_{2} \ldots<i_{M}$. Again this subspace can be mapped into the one for hard-core bosons on the full chain $\mathcal{L}$ and the effective low-energy Hamiltonian is again a $t-V$ model with $t=J_{\perp}, V=J_{z}$ and $\mu=B-D$. Since $V \gg t$, the IS2 phase $(M=L / 2)$ remains stable over a broad region of field. However, as shown in Fig.2, a second order transition to a spin liquid (SL) phase occurs at the critical value $B=B_{c 3}$ that is obtained from the exact solution of the Bethe-Ansatz equations for the $t-V$ model [17]:

$B_{c 3}=D+J_{\perp} \Delta+\frac{4 \pi J_{\perp} \sinh \gamma}{\gamma} \sum_{n=0}^{\infty} \frac{1}{\cosh \left[(2 n+1) \pi^{2} / 2 \gamma\right]}$

where $\Delta=2 \cosh \gamma$. The three transitions at $B_{c 1}, B_{c 2}$ and $B_{c 3}$ belong to the Dzhaparidze-Nersesyan-PokrovskyTalapov universality class [18]. Finally, the system becomes fully saturated at the critical field $B_{c 4}=2 J_{z}+2 J_{\perp}+D$ (this expression is also valid away from the strongly anisotropic limit $\Delta \gg 1$ ).

In order to extend the quantum phase diagram of $H$ away from the strongly anisotropic limit, we have used the Stochastic Series expansion (SSE) [19] quantum Monte Carlo (QMC) method. The simulations were done on finite chains of length $16 \leq L \leq 64$. The SSE is a finite-temperature QMC based on the Taylor expansion of the partition function, $e^{-\beta H}$. Ground
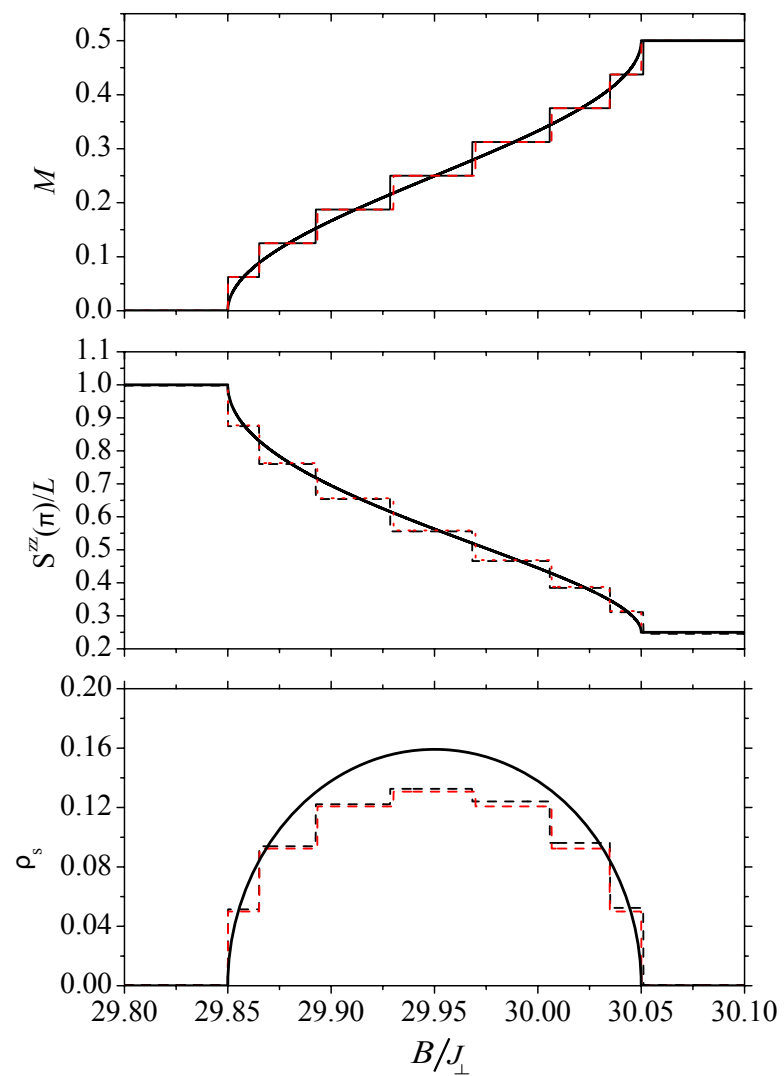

FIG. 1: (Color online) Magnetization along the field direction $M_{z}$ (a), longitudinal structure factor $S^{z z}(\pi) / L(\mathrm{~b})$, and spin stiffness $\rho_{s}$ (c) as a function of field for $J_{z}=20 J_{\perp}$ and $D=10 J_{\perp}$. The dotted (dashed) line corresponds to the exact solution of the original (effective) model in a 16 sites chain. The full line corresponds to the exact solution of the effective model in the thermodynamic limit $L \rightarrow \infty$.

state estimates for the observables are obtained by choosing sufficiently large values of the inverse temperature $\beta$. For the parameters explored in this study, $\beta=2 L$ was found to be sufficient for the observables to have converged to their ground state values. The so-called Haldane state is the only new phase that appears in the full quantum phase diagram (see Fig 2) relative the strongly anisotropic limit. To characterize the different emergent phases, we computed $M, \rho_{s}$ and $S^{z z}(q)$. The spin stiffness, $\rho_{s}$, is simply obtained by computing the winding number $(W)$ fluctuations of the world lines: $\rho_{s}=\left\langle W^{2}\right\rangle / \beta$. [20]

Both Ising phases, IS1 and IS2, are marked by a finite value of $S^{z z}(Q) \propto L$ and a vanishing value of $\rho_{s}$ in the limit $L \rightarrow \infty$. The spin SS phase is characterized by a finite value of both $S^{z z}(Q) / L$ and $\rho_{s}$ in the same limit, while only $\rho_{s}$ remains finite $\left(S^{z z}(Q) / L \rightarrow 0\right)$ for the SL phase. Finally, the Haldane phase is characterized by a hidden ordering [21] and both quantities, $\rho_{s}$ and $S^{z z}(Q) / L$ go to zero in the thermody- 
namic limit. Since all these quantities are finite for finite size systems and estimates for $L \rightarrow \infty$ are obtained from finitesize scaling.

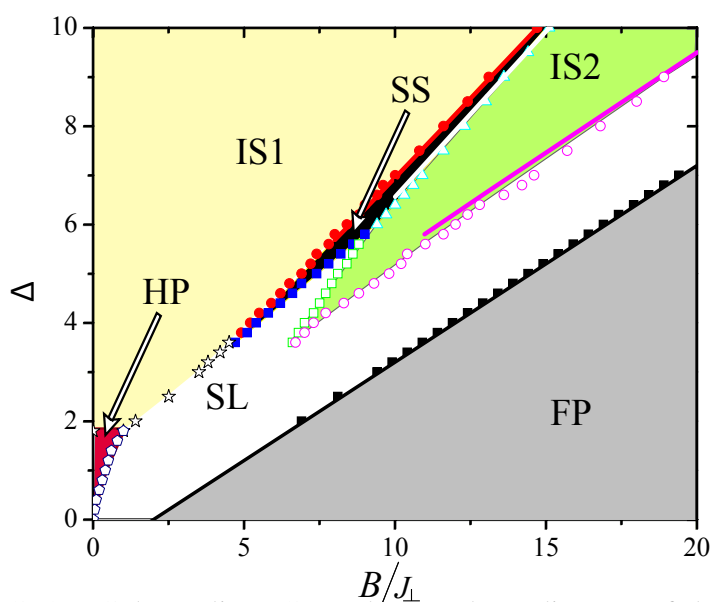

FIG. 2: (Color online) Ground state phase diagram of the $S=1$ Heisenberg model in one dimension in the $\Delta-B$ parameter space with $D=\Delta / 2$. The spin-gapped Ising-like phases IS1 $\left(m_{z}=\right.$ $0)$ and IS2 $\left(m_{z}=0.5\right)$ phases have long range diagonal order $\left(S^{z z} / L>0\right.$ for $\left.L \rightarrow \infty\right)$ whereas the SL phase has a finite stiffness $\left(\rho_{s}>0\right)$. The spin SS has simultaneous long range diagonal order and finite stiffness while both quantities are zero in the Haldane phase (HP). The symbols are results obtained from QMC simulations. The solid lines are the phase boundaries, $B_{c i}$ with $1 \leq i \leq 4$ (see Eqs 15 and 16 obtained from the effective low-energy models valid for $\Delta \gg 1$.

The results of the simulation are summarized in the ground state phase diagram for the $\Delta-B$ parameter space with $D=\Delta / 2$ (Fig 2). For $\Delta \leq 1$, the ground state is a SL with finite $\rho_{s}$ above a critical field $B_{c 0}$ and up to the saturation field $B_{c 4}$. Below $B_{c 0}$, the ground state is in the Haldane phase [10]. If we increase $\Delta$, we find that the Haldane phase (HP) is separated from the IS1 phase by a line of critical points. This line appears because the excitations that becomes gapless at $\Delta=\Delta_{c} \simeq 1.8$ [22] and $B=0$ have $M=0$ (this is a second order transition between two $M=0$ ground states) while the $|M|>1$ excitations remain gapped. The Ising-like phase IS1 evolves continuously into the state $\left|\psi_{\mathcal{A}}^{0}\right\rangle$ (or $\left|\psi_{\mathcal{B}}^{0}\right\rangle$ ) for $\Delta \gg 1$ (see Eq 2). This corresponds to the empty band of the effective model $\tilde{H}_{\mathcal{A}}$. For $\Delta \lesssim 3.6$, increasing the field $B$ induces a transition from the IS1 phase to the SL and from the SL to the fully polarized (FP) phase. At larger $\Delta(\gtrsim 3.6)$, the Ising ordering persists along with the SL for a finite range of $B$ giving rise to a spin SS that is continuously connected with the SS phase that was analytically obtained in the limit $\Delta \gg 1$. For $\Delta \gtrsim 5.8$, the SS phase ends up in the IS2 phase, as obtained for $\Delta \gg 1$, while the SL phase appears in between for $3.6 \lesssim \Delta \lesssim 5.8$. At even higher fields, there is an IS2SL transition at $B=B_{c 3}$ and the system reaches saturation $(M / L=1)$ for $B=B_{c 4}$. Both phases and transitions are continuously connected with the ones obtained for $\Delta \gg 1$. The phase boundaries, $B_{c i}$ with $1 \leq i \leq 4$, obtained from the low energy effective models are shown in Fig 2 with solid lines. The close agreement with numerics for $\Delta \gg 1$ confirms the validity of the low energy models in this limit.

In summary, we have demonstrated the existence of a spin SS phase induced by field in a one-dimensional Heisenberg model with strong uniaxial anisotropy. This demonstration can be easily extended to higher dimensions [6]. We have also computed the quantum phase diagram as a function of the exchange anisotropy $\Delta$ and field $B$. In particular, our ground state solution becomes asymptotically exact in the strongly anisotropic limit allowing for a full characterization of the SS spin phase. This is a remarkable result considering that SS phases found in other models were always obtained from numerical or approximated treatments.

We thank B. S. Shastry and T. Giamarchi for helpful discussions. LANL is supported by US DOE under Contract No. W-7405-ENG-36.

[1] E. Kim and M. H. W. Chan, Nature 427225 (2004); Science 305, 1941 (2004).

[2] O. Penrose and L. Onsager, Phys. Rev. 104, 576 (1956).

[3] M. Boninsegni and N. Prokofe'v, Phys. Rev. Lett 95, 237204 (2005); S. Wessel and M. Troyer, Phys. Rev. Lett 95, 127205 (2005); D. Heidarian and K. Damle ibid 95, 127206 (2005); R. G. Melko et al., ibid 95127207 (2005).

[4] P. Sengupta, L. P. Pryadko, F. Alet, M. Troyer and G. Schmid, Phys. Rev. Lett. 94, 207202 (2005).

[5] G. G. Batrouni, F. Hebert, R. T. Scalettar, Phys. Rev. Lett. 97, 087209 (2006).

[6] P. Sengupta and C. D. Batista, Phys. Rev. Lett. 98, 227201 (2007).

[7] K-K Ng and T. K. Lee, Phys. Rev. Lett. 97, 127204 (2006).

[8] N. Laflorencie and F. Mila, Phys. Rev. Lett. 99, 027202 (2007).

[9] K. P. Schmidt, A. M. Laeuchli and F. Mila, arXiv:0706.1517.

[10] F. D. M. Haldane, Phys. Lett. 93A, 464 (1983); 50A, 1153 (1983); 57A, 3359 (1985).

[11] E. Polizzi, F. Mila and E. S. Sorensen, Phys. Rev. B 58, 2407 (1998); C. D. Batista, K. Hallberg and A. A. Aligia, Phys. Rev. B 58, 9248 (1998).

[12] See for instance T. Giamarchi, Quantum Physics in One Dimension, (Oxford Science Publications, 2004).

[13] C. N. Yang. and C. P. Yang, Phys. Rev. 150, 327 (1966).

[14] T. Matsubara and H. Matsuda, Prog. Theor. Phys. 16, 569 (1956).

[15] C. D. Batista and G. Ortiz, Phys. Rev. Lett. 86, 1082 (2001).

[16] C. D. Batista and G. Ortiz, Adv. 53, 1 (2004).

[17] J. des Cloizeaux and M. Gaudin, J. Math. Phys. 7, 1384 (1966).

[18] G. I. Dzhaparidze and A. A. Nersesyan, JETP Lett. 27, 334 (1978); V. L. Pokrovsky and A. L. Talapov, Phys. Rev. Lett. 42, 65 (1979); D. C. Cabra, A. Honecker and P. Pujol, Phys. Rev. B 58, 6241 (1998).

[19] A. W. Sandvik, Phys. Rev. B 59, R14157 (1999).

[20] E. L. Pollock, and D. M. Ceperley, Phys. Rev. B 36, 8343 (1987).

[21] M. den Nijs and K. Rommelse, Phys. Rev. B 40, 4709 (1989).

[22] W. Chen, K. Hida and B. C. Sanctuary, Phys. Rev. B 67, 104401 (2003). 